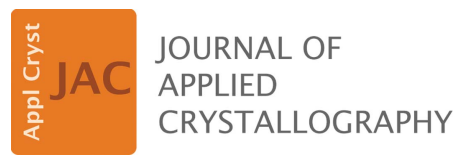

ISSN $1600-5767$

Received 2 August 2019

Accepted 29 November 2019

Edited by G. J. McIntyre, Australian Nuclear Science and Technology Organisation, Lucas Heights, Australia

Keywords: neutron Larmor diffraction; neutron spin-echo; polycrystalline samples; powder diffraction.

Supporting information: this article has supporting information at journals.iucr.org/j

\section{Neutron Larmor diffraction on powder samples}

\author{
Thomas Keller, ${ }^{\mathrm{a}, \mathrm{b}} *$ Piotr Fabrykiewicz, ${ }^{\mathrm{c}}$ Radosław Przeniosło, ${ }^{\mathrm{c}}$ Izabela Sosnowska ${ }^{\mathrm{c}}$ \\ and Bernhard Keimer ${ }^{\mathrm{a}}$ \\ ${ }^{\mathbf{a}}$ Max Planck Institute for Solid State Research, Stuttgart, Germany, ${ }^{\mathbf{b}}$ Max Planck Society Outstation at the \\ Forschungsneutronenquelle Heinz Maier-Leibnitz (FRM-II), Garching, Germany, and ${ }^{\mathbf{c}}$ Faculty of Physics, University of \\ Warsaw, Poland. *Correspondence e-mail: t.keller@fkf.mpg.de
}

A hitherto unrecognized resolution effect in neutron Larmor diffraction (LD) is reported, resulting from small-angle neutron scattering (SANS) in the sample. Small distortions of the neutron trajectories by SANS give rise to a blurring of the Bragg angles of the order of a few hundredths of a degree, leading to a degradation of the momentum resolution. This effect is negligible for single crystals but may be significant for polycrystalline or powder samples. A procedure is presented to correct the LD data for the parasitic SANS. The latter is accurately determined by the SESANS technique (spin-echo small-angle neutron scattering), which is readily available on Larmor diffractometers. The analysis technique is demonstrated on LD and SESANS data from $\alpha-\mathrm{Fe}_{2} \mathrm{O}_{3}$ powder samples. The resulting $d$-spacing range agrees with experimental data from high-resolution synchrotron radiation powder diffraction on the same sample.

\section{Introduction}

Neutron Larmor diffraction (LD) is a high-resolution technique which permits the measurement of lattice spacings $d_{h k l}$ and their range $\Delta d_{h k l}$. The latter arises, for example, from microstrains, magnetostriction, or structural and magnetic domains, or from a small splitting of Bragg peaks resulting from distortions of the crystal lattice. The resolution of current Larmor diffractometers is $\sim 10^{-6}$ (relative) of the lattice spacing. Further improvement by one order of magnitude is possible by an optimized instrument design.

The present work is related to a review of the symmetry properties and subsequent revision of the crystal structures of numerous compounds. Synchrotron radiation (SR) powder diffraction studies of the multiferroic $\mathrm{BiFeO}_{3}$ (Sosnowska et al., 2012), $\mathrm{CaCO}_{3}$, calcite (Przeniosło et al., 2016), the transition metal oxides $\mathrm{V}_{2} \mathrm{O}_{3}, \mathrm{Al}_{2} \mathrm{O}_{3}$ and $\mathrm{Cr}_{2} \mathrm{O}_{3}$ (Fabrykiewicz et al., 2018), and hematite $\left(\alpha-\mathrm{Fe}_{2} \mathrm{O}_{3}\right)$ (Przeniosło et al., 2014) have shown monoclinic symmetry instead of the previously established trigonal one. Similar studies have shown distortions of the cubic symmetry of chromium (Przeniosło et al., 2018) and $\mathrm{MnO}$ (Fabrykiewicz \& Przeniosło, 2016). The monoclinic distortion observed in $\alpha-\mathrm{Fe}_{2} \mathrm{O}_{3}$ is of the order of $\Delta d / d \simeq$ $2 \times 10^{-4}$, i.e. close to the detection limit of SR powder diffraction methods. The LD experiments in this work were conducted in order to confirm this small lattice distortion with a complementary technique and thus to validate the SR data.

LD is based upon the Larmor precession of the neutron spins in parallelogram-shaped magnetic fields along the incident and scattered beams. The geometry of these fields, sometimes labelled precession devices (PDs), was first 
proposed by Rekveldt (Rekveldt, 2000; Rekveldt et al., 2001). Tuning of the field boundaries of the PDs parallel to the diffracting lattice planes yields a Larmor precession phase $\phi_{\mathrm{LD}} \propto d_{h k l}$, where $\phi_{\mathrm{LD}}$ is decoupled from the spectral width and collimation of the neutron beam. The characteristic limitation of conventional diffractometers imposed by the inverse proportionality between intensity and resolution is not effective in LD, and so excellent momentum resolution without excessive loss of intensity is achieved. In this respect, LD is similar to neutron spin-echo, where the energy resolution is decoupled from the monochromaticity of the incident neutron beam.

Experimental studies taking advantage of LD include thermal expansion under hydrostatic and uniaxial pressure (Pfleiderer et al., 2007; Niklowitz et al., 2010; Bourdarot et al., 2011), the formation of structural and antiferromagnetic domains (Náfrádi et al., 2016), and the splitting of Bragg peaks by lattice distortions (Inosov et al., 2013; Hu et al., 2015; Wang et al., 2018). For thermal expansion only relative changes in the lattice constants are relevant, but LD can also provide absolute values of $d_{h k l}$ when the instrument is calibrated with a reference monocrystal, such as silicon or germanium. In this way, $d_{h k l}$ values for polycrystalline Inconel alloys were accurately measured to determine residual stresses (Repper et al., 2010), and in spin-ice materials the exact values of the lattice constants allowed quantification of the emergent magnetic monopole charge (Ruminy et al., 2016).

The Larmor diffractometers TRISP at the Heinz MaierLeibnitz Zentrum (Keller et al., 2002; Keller \& Keimer, 2015), FLEXX at the Helmholtz-Zentrum Berlin (Groitl et al., 2015) and ZETA at the Institut Laue-Langevin (Martin et al., 2012) were originally designed as three-axis spin-echo spectrometers optimized for the spectroscopy of phonons and spin excitations. These instruments take advantage of the resonant neutron spin-echo technique (NRSE) to define the parallelogram-shaped field regions by radio frequency (RF) spin-flip coils (Golub \& Gähler, 1987; Gähler \& Golub, 1988). A recent development uses Wollaston prisms, triangular-shaped DC magnetic fields bounded by superconducting sheets ( $\mathrm{Li}$ et al., 2017), as PDs. The advantages of the latter technique are the compact geometry and the increased flexibility achieved by tuning the inclination angles of the magnetic fields by electrical currents, instead of mechanical rotation as in the case of NRSE.

The resolution of LD depends only weakly on the sample mosaic, and for polycrystalline samples no significant reduction in the resolution compared with single crystals is expected. This was confirmed experimentally on polycrystalline aluminium (Rekveldt et al., 2001) and Inconel alloy (Repper et al., 2010). If the size of the crystallites is in the submicrometre range, small-angle neutron scattering (SANS) of the incident and diffracted beams might lead to a blurring of the Bragg angles. This effect is typically small, with SANS angles of the order of a few hundredths of a degree, and there will be no SANS intensity at the large Bragg angles $\theta_{\mathrm{B}} \simeq 50^{\circ}$ used in LD experiments. Initial LD measurements performed with $\alpha-\mathrm{Fe}_{2} \mathrm{O}_{3}$ polycrystalline samples in cylindrical vanadium containers have shown $d$-spacing ranges considerably broader than those obtained with SR diffraction. This broadening results from small-angle scattering in the sample, which is negligible in the SR experiments, as it increases with sample diameter and neutron or X-ray wavelength. Both parameters are more favourable in the case of SR, with a sample diameter (capillary) of $0.5 \mathrm{~mm}$ and an X-ray wavelength of $0.4 \AA$, compared with a sample width of several millimetres and a neutron wavelength of $\sim 2 \AA$ in LD.

In this paper we discuss the resolution of LD including SANS. The effect can be corrected for, if the SANS scattering probability $S(\theta)$ is known. Calculation of $S(\theta)$ is possible, but it requires detailed knowledge of the powder grain morphology and scattering length density profiles. In addition, strong multiple scattering is expected for the large sample diameters of several millimetres typically used in neutron diffraction experiments. Accurate modelling of this multiple scattering is difficult. Thus, a measurement of $S(\theta)$ is preferable. This can be efficiently performed on the Larmor diffractometer, which is readily converted into a spin-echo small-angle scattering (SESANS) instrument by changing the field polarities and inclination angles of the PDs (Rekveldt, 1996; Rekveldt et al., 2003; Keller et al., 1995). Both LD and SESANS data are then consistently collected on the same sample in one experimental run.

In the following we first review LD including parasitic SANS. We discuss the properties of the SESANS technique and derive a method for correcting LD data for SANS effects. Finally, we work out an example of the analysis technique using experimental data from $\alpha-\mathrm{Fe}_{2} \mathrm{O}_{3}$ powder samples.

\section{Larmor diffraction including SANS}

In this section we review the principles of $L D$ and then include the effect of SANS.

Rekveldt proposed the field geometry shown in Fig. 1, a uniform DC magnetic field $\mathbf{B}_{0}$ with boundaries oriented parallel to the diffracting lattice planes (Rekveldt, 2000; Rekveldt et al., 2001). The neutron spins are initially polarized perpendicular to $\mathbf{B}_{0}$ and cross the field twice, before and after being diffracted at the lattice planes of a crystal with interplanar spacing $d_{h k l}$ and corresponding reciprocal-lattice vector $G=2 \pi / d_{h k l}$. The Larmor phase $\phi_{\mathrm{LD}}=\omega_{\mathrm{L}} t_{\mathrm{t}}$ accumulated by a neutron spin after passing both fields is proportional to the Larmor frequency $\omega_{\mathrm{L}}=\gamma B_{0}$, with $\gamma=2 \pi \times 2.916 \mathrm{kHz}$ gauss $^{-1}$ and the transition time $t_{\mathrm{t}}=L / v_{\perp}$ that the neutron spends in the field. The parameter $v_{\perp}$ is the velocity component perpendicular to the field boundary, with $v_{\perp}=(h / m) k_{\perp}, m$ the neutron mass and $k_{\perp}=G / 2=\pi / d_{h k l}$ :

$$
\phi_{\mathrm{LD}}=\omega_{\mathrm{L}} \frac{2 L}{v_{\perp}}=\frac{2 m}{\pi \hbar} \omega_{\mathrm{L}} L d_{h k l} .
$$

$\phi_{\mathrm{LD}}$ only depends on the field integral $J_{0}=2 \omega_{\mathrm{L}} L$ and the lattice spacing $d_{h k l}$, and is independent of the Bragg angle. The resonant spin-echo technique offers an easy way of defining the required flat-field boundaries by radio-frequency spin-flip coils (Fig. 1). 
A shift in $d_{h k l}$, for example by thermal expansion, is measured by tracking the phase shift $\Delta \phi(T)=\phi_{\mathrm{LD}} \epsilon_{h k l}$ versus the temperature $T$, where

$$
\epsilon_{h k l}=d_{h k l} / d_{h k l}^{\circ}-1 .
$$

$d_{h k l}^{\circ}$ is the mean or unperturbed value of $d_{h k l} . d_{h k l}$ is typically spread, for example by the effect of microstrains. Then the phase is also spread as

$$
\Delta \phi_{\mathrm{LD}}=\phi_{\mathrm{LD}}^{\circ} \epsilon_{h k l} .
$$

$\phi_{\mathrm{LD}}^{\circ}$ is related to $d_{h k l}^{\circ}$ via equation (1). The final beam polarization is given by the average

$$
\begin{aligned}
P_{\mathrm{LD}}\left(\phi_{\mathrm{LD}}\right) & =\left\langle\cos \left(\Delta \phi_{\mathrm{LD}}\right)\right\rangle \\
& =\int D\left(\epsilon_{h k l}\right) \cos \left(\phi_{\mathrm{LD}} \epsilon_{h k l}\right) \mathrm{d} \epsilon_{h k l} .
\end{aligned}
$$

$D\left(\epsilon_{h k l}\right)$ is a normalized distribution function. $P\left(\phi_{\mathrm{LD}}\right)$ is measured for a series of values $\phi_{\mathrm{LD}}\left(B_{0}\right)$, where for each $\phi_{\mathrm{LD}}$ the position $\Delta L$ of coil $\mathrm{C} 4$ is scanned through one Larmor period as sketched in Fig. 1. $P$ is then obtained from the amplitude of the cosine-shaped $I(\Delta L)$. (The integration limits of all integrals in this section can be chosen as $\mp \infty$.)

For the measurement of thermal expansion it is important to note that $\Delta \phi(T)$ is affected by $D\left(\epsilon_{h k l}\right)$, if the latter is asymmetric and if the asymmetry changes with $T$. This might result from an asymmetric splitting of a Bragg peak related to a structural phase transition. In cases where $D\left(\epsilon_{h k l}\right)$ is symmetric, even if the width or shape changes with $T$, there will be no effect on $\Delta \phi(T)$.

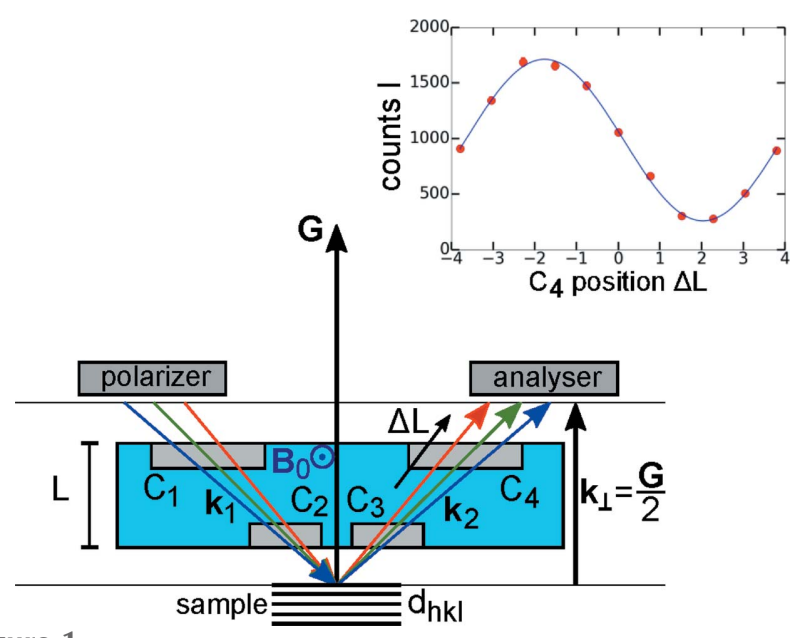

Figure 1

Sketch of a Larmor diffractometer. A polarized neutron beam crosses the uniform field $\mathbf{B}_{0}$ twice. The boundaries of $\mathbf{B}_{0}$ are oriented parallel to the lattice planes $d_{h k l}, G=2 \pi / d_{h k l}$. In the resonant spin-echo configuration (NRSE), instead of a uniform field $\mathbf{B}_{0}$ four RF spin flippers $\mathrm{C}_{1}-\mathrm{C}_{4}$ define the boundaries of the precession regions, and there is no field along the flight path between the flippers and in the sample region. $\mathrm{C} 1-\mathrm{C} 4$ are double coils, each consisting of two single RF spin-flip coils with opposite field direction (known as the 'bootstrap' technique; Gähler \& Golub, 1988). The Larmor phase shift $\Delta \phi_{\mathrm{LD}}$ and the polarization are obtained from the count rate $I$ versus the position $\Delta L$ of the coil $\mathrm{C}_{4}(\Delta L$ in the inset is in units of millimetres): $I(\Delta L)=I_{0}\left\{1+P \cos \left[2 \pi\left(\Delta L-\Delta L_{0}\right) /\right.\right.$ $\left.\left.L_{\mathrm{per}}\right]\right\}$, where the period is $L_{\mathrm{per}}=2 \pi v_{\mathrm{n}} / \omega_{\mathrm{L}}, v_{\mathrm{n}}$ is the neutron velocity and $\omega_{\mathrm{L}}$ the Larmor frequency. With the offset $\Delta L_{0}$, the phase is $\Delta \phi_{\mathrm{LD}}=$ $2 \pi \Delta L_{0} / L_{\text {per }}$.
We now include the distortion of the neutron trajectories by SANS, and the misalignment between the lattice planes and the boundaries of $\mathbf{B}_{0}$, which necessarily happens for mosaic or polycrystalline samples. Both effects disturb the correlation between the lattice vector $G$ and $k_{\perp}$, such that $k_{\perp} \neq G / 2$. This distortion of the trajectories by SANS leads to an accumulation of additional Larmor phase, resulting in a reduction in $P$ in equation (4). The calculation of the phase change depends on whether SANS happens before or after Bragg diffraction. SANS in the incident beam changes the Bragg angle, whereas the path length in the first precession field stays the same, so that the Larmor phase changes. SANS in the diffracted beam does not affect the Bragg angle, but the path length in the second precession field changes.

We first calculate $\phi_{\mathrm{LD}}$ for single SANS events in the incident and diffracted beams, and then take the average assuming a range of SANS angles, which will be quantitatively determined in a separate SESANS experiment. The geometry is defined in Fig. 2, with the following relations:

$$
\begin{aligned}
& \theta_{1}=\theta_{0}-\alpha_{1}+\eta, \\
& \theta_{2}=\theta_{1}+\alpha_{2}+\eta, \\
& L_{1}=L / \sin \left(\theta_{0}\right), \\
& L_{2}=L / \sin \left(\theta_{2}\right), \\
& \lambda=2 d_{h k l} \sin \left(\theta_{1}\right),
\end{aligned}
$$

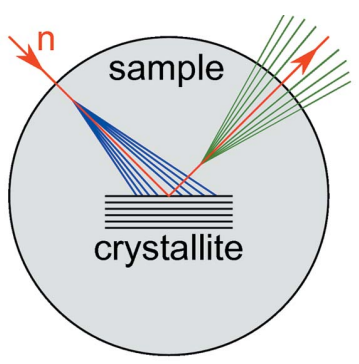

(a)

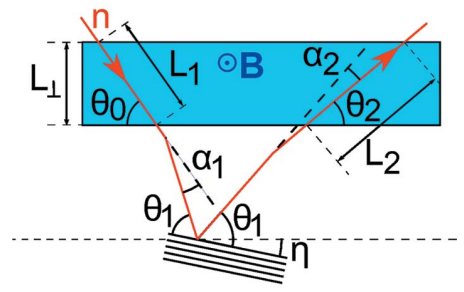

(b)

Figure 2

(a) SANS in a powder sample before and after Bragg diffraction at a crystallite. (b) A single Bragg diffraction event at a crystallite. $L_{1,2}$ are the path lengths in the precession field $\mathbf{B} . L$ is the width of the field area $\mathbf{B}_{0}$. $\alpha_{1,2}$ are the SANS angles. $\theta_{0}$ and $\theta_{2}$ are the angles of the trajectories with the field boundaries, and $\theta_{1}$ is the Bragg angle. $\eta$ is the angle between the field boundary and the diffracting lattice plane. The signs of $\theta_{0}, \theta_{1}$ and $\theta_{2}$ are positive. $\alpha_{1}, \alpha_{2}$ and $\eta$ have a negative sign for clockwise rotation, as shown in the sketch. 


$$
\begin{gathered}
v=\frac{\pi \hbar}{m d_{h k l} \sin \left(\theta_{1}\right)}, \\
d_{h k l}=d_{h k l}^{\circ}\left(1+\epsilon_{h k l}\right) .
\end{gathered}
$$

[The last equation is identical to equation (2) and is repeated here for better readability.] $m$ and $v=\hbar k / m$ are the neutron mass and velocity, respectively. The Larmor phases $\phi_{1,2}$ accumulated before and after Bragg diffraction are

$$
\phi_{1,2}=\frac{\omega_{\mathrm{L}} L_{1,2}}{v} .
$$

The total phase is

$$
\begin{aligned}
\phi_{\mathrm{LD}} & =\phi_{1}+\phi_{2} \\
& =\frac{\omega_{\mathrm{L}}\left(L_{1}+L_{2}\right)}{v} \\
& =\frac{m}{\pi \hbar} \omega_{\mathrm{L}} L d_{h k l}^{\circ}\left(1+\epsilon_{h k l}\right)\left[\frac{\sin \left(\theta_{1}\right)}{\sin \left(\theta_{0}\right)}+\frac{\sin \left(\theta_{1}\right)}{\sin \left(\theta_{2}\right)}\right] .
\end{aligned}
$$

Expanding equation (7) with the definitions of equations (5) to first order in $\epsilon, \alpha_{1,2}$ and $\eta$ gives

$$
\Delta \phi_{\mathrm{LD}}=\phi_{\mathrm{LD}}^{\circ}\left[\epsilon_{h k l}-\frac{\alpha_{1}+\alpha_{2}}{2} \cot \left(\theta_{0}\right)\right]
$$

where

$$
\begin{gathered}
\phi_{\mathrm{LD}}^{\circ}=\phi_{\mathrm{LD}}\left(\epsilon_{h k l}=0, \theta_{1}=\theta_{2}=\theta_{0}\right), \\
\Delta \phi_{\mathrm{LD}}=\phi_{\mathrm{LD}}-\phi_{\mathrm{LD}}^{\circ} .
\end{gathered}
$$

The first-order term in $\eta$ is zero. The second-order terms of the preceding expansion are summarized in Appendix $A$. The leading term is $\sim \cot ^{2}\left(\theta_{0}\right) \eta^{2}$, which is negligible for the experimental parameters in this work. The first term in equation (8) is the usual LD phase related to variations in $d_{h k l}$, while the second term describes the small-angle scattering.

The polarization $P\left(\phi_{\mathrm{LD}}\right)$ is given as a generalization of equation (4) by the average

$$
\begin{aligned}
P\left(\phi_{\mathrm{LD}}^{\circ}\right)= & \left\langle\cos \left(\Delta \phi_{\mathrm{LD}}\right)\right\rangle \\
= & \iiint D\left(\epsilon_{h k l}\right) S\left(\alpha_{1}\right) T_{1}\left(\alpha_{1}\right) S\left(\alpha_{2}\right) T_{2}\left(\alpha_{2}\right) \\
& \times \cos \left\{\phi_{\mathrm{LD}}^{\circ}\left[\epsilon_{h k l}-\frac{\alpha_{1}+\alpha_{2}}{2} \cot \left(\theta_{0}\right)\right]\right\} \mathrm{d} \alpha_{1} \mathrm{~d} \alpha_{2} \mathrm{~d} \epsilon_{h k l} .
\end{aligned}
$$

$S\left(\alpha_{1}\right)$ and $S\left(\alpha_{2}\right)$ are the normalized ranges of the SANS angles before and after scattering, respectively, and $T_{1,2}\left(\alpha_{1,2}\right)$ are angular transmission functions of the diffractometer, given by the collimations of the incident and scattered beams, respectively. The cosine term in equation (11) splits according to $\cos (a+b+c)=\cos (a) \cos (b) \cos (c)+\sin \ldots$, and the sine terms integrate to zero, as the $S$ and $T$ distribution functions are symmetric. The polarization is then

$$
\begin{gathered}
P\left(\phi_{\mathrm{LD}}^{\circ}\right)=P_{S 1} P_{S 2} P_{\mathrm{LD}}, \\
P_{S 1}=\int S_{1}\left(\alpha_{1}\right) T_{1}\left(\alpha_{1}\right) \cos \left[\phi_{\mathrm{LD}}^{\circ} \cot \left(\theta_{0}\right) \alpha_{1} / 2\right] \mathrm{d} \alpha_{1},
\end{gathered}
$$

$$
P_{S 2}=\int S_{2}\left(\alpha_{2}\right) T_{2}\left(\alpha_{2}\right) \cos \left[\phi_{\mathrm{LD}}^{\circ} \cot \left(\theta_{0}\right) \alpha_{2} / 2\right] \mathrm{d} \alpha_{2},
$$

where $P_{\mathrm{LD}}$ is given by equation (4). The first two factors, $P_{S 1}$ and $P_{S 2}$, describe the effect of SANS before and after diffraction, respectively. These factors differ in the beam collimation $T_{1,2}$ and the width of the small-angle scattering $S_{1,2}$. The widths of $S_{1,2}$ will typically be much smaller than the widths of $T_{1,2}$, such that the latter have only a small influence on the value of the integral. Thus we will simplify $T_{1}=T_{2}=T$. The widths of $S_{1}$ and $S_{2}$ are only identical for symmetric scattering configurations, such as cylindrical sample cross sections, or flat samples in a symmetric configuration with respect to $k_{1,2}$, and might be quite different for asymmetric sample shapes.

One aim of an LD experiment is to measure the distribution width of the lattice spacing $D\left(\epsilon_{h k l}\right)$. To extract $P_{\mathrm{LD}}$ from the experimental data $P\left(\phi_{\mathrm{LD}}^{\circ}\right)$ in equation (12), the SANS integrals $P_{S 1}, P_{S 2}$ have to be known. In the following section we show that $P_{S}$ can be determined experimentally by taking advantage of the SESANS technique, and thus for the data analysis there is no need to use an analytical expression for $P_{S}$. Nevertheless, an analytical formula is often helpful. As a simple model, we take Gaussians for the distributions $D, S_{1,2}$ and $T$, with FWHMs of $\epsilon_{\mathrm{FW}}, \alpha_{S 1,2}$ and $\alpha_{T}$, respectively. These Gaussians have the form

$$
\exp \left[\frac{-4 \ln (2) x^{2}}{x_{\text {FWHM }}^{2}}\right]
$$

Using the tabulated integral

$$
\int_{-\infty}^{+\infty} \exp \left(-a^{2} x^{2}\right) \cos (b x) \mathrm{d} x=\left(\frac{\pi}{a^{2}}\right)^{1 / 2} \exp \left(\frac{-b^{2}}{4 a^{2}}\right),
$$

we obtain for the polarization [equation (12)]

$$
\begin{gathered}
P_{\mathrm{LD}}=\exp \left(-C_{1} \epsilon_{h k l \mathrm{FW}}^{2}\right) \\
P_{S 1}=\exp \left(\frac{-C_{2}}{\alpha_{S 1}^{-2}+\alpha_{T}^{-2}}\right) \\
P_{S 2}=\exp \left(\frac{-C_{2}}{\alpha_{S 2}^{-2}+\alpha_{T}^{-2}}\right), \\
C_{1}=\frac{\left(\phi_{\mathrm{LD}}^{\circ}\right)^{2}}{16 \ln (2)}, \\
C_{2}=\frac{\left(\phi_{\mathrm{LD}}^{\circ}\right)^{2}}{16 \ln (2)} \frac{\cot ^{2}\left(\theta_{0}\right)}{4} .
\end{gathered}
$$

\section{The SESANS technique}

We briefly review the principles of SESANS and give scaling formulas for flat and cylindrical samples to convert SESANS data to the LD geometry. SESANS uses two identical field regions (or precession regions defined by RF spin flippers) 
with opposite signs of the magnetic field, with boundaries inclined by an angle $\Omega$ (Fig. 3). Unscattered neutrons gain equal precession phases in both regions $\left(\phi_{1}=-\phi_{2}\right)$, even if the path is inclined with respect to the central axis. If the neutron is scattered by a small angle $\alpha$, the path length in the second field changes (Rekveldt, 1996; Rekveldt et al., 2003; Keller et al., 1995):

$$
\Delta L=L_{0} \tan (\Omega) \alpha .
$$

The phase difference $\Delta \phi=\phi_{1}-\phi_{2}$ is

$$
\Delta \phi=\phi_{1} \tan (\Omega) \alpha
$$

where $\phi_{1}$ is the Larmor phase accumulated in one precession region,

$$
\phi_{1}=\frac{m \omega_{\mathrm{L}} L_{0}}{\hbar k} .
$$

The polarization $P\left(\phi_{1}\right)$ is given by an integral similar to $P_{S 1}$, $P_{S 2}$ in equation (12):

$$
\begin{aligned}
P_{\mathrm{SES}}\left(\phi_{1}\right) & =\langle\cos (\Delta \phi)\rangle \\
& =\int S(\alpha) T(\alpha) \cos \left[\phi_{1} \tan (\Omega) \alpha\right] \mathrm{d} \alpha .
\end{aligned}
$$

It is not necessary to model $S(\alpha)$ for subsequent analysis of the LD data, but it is often convenient to have an analytical expression. As a simple model we again take Gaussian distributions [equation (15)] for $S(\alpha)$ and $T(\alpha)$ with FWHMs of $\alpha_{S}$ and $\alpha_{T}$, respectively. Using equation (16) we get

$$
P_{\mathrm{SES}}\left(\phi_{1}\right)=\exp \left[-\frac{\phi_{1}^{2} \tan ^{2}(\Omega)}{16 \ln (2)\left(\alpha_{S}^{-2}+\alpha_{T}^{-2}\right)}\right] .
$$

Typically $\alpha_{S} \ll \alpha_{T}$, so that the latter is negligible in equation (26).

It is useful to express the SESANS phase [equation (23)] in terms of the spin-echo length $z$ and the momentum transfer $Q$ :

$$
\Delta \phi=z Q
$$

where

$$
z=\frac{\phi_{1} \tan (\Omega)}{k}
$$

or

$$
\begin{aligned}
z(\AA)= & 0.2531\left(\mathrm{~m}^{-1} \mathrm{kHz}^{-1} \AA^{-1}\right) \tan (\Omega) \times L_{0}(\mathrm{~m}) \\
& \times v_{L}(\mathrm{kHz}) \times \lambda^{2}\left(\AA^{2}\right),
\end{aligned}
$$

with $v_{L}=\omega_{L} /(2 \pi)$.

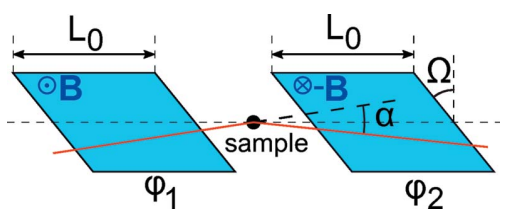

Figure 3

Spin-echo small-angle scattering configuration. The fields $B$ of the two precession regions have opposite signs, as opposed to the LD configuration.
We now show how $P_{\mathrm{SES} \text { cyl }}$ for a cylindrical sample can be calculated using SESANS data $P_{\text {SES flat }}$ from a flat sample, and vice versa. We will take advantage of a scaling relation for $P_{\mathrm{SES}}$ with respect to $k$ and the sample width $t$ derived by Rekveldt and Andersson (Rekveldt et al., 2003; Andersson et al., 2008), which is valid even in the presence of strong multiple scattering:

$$
P_{\mathrm{SES}}(z, k, t)=P_{\mathrm{SES}}\left(z, k_{0}, t_{0}\right)^{\left(k_{0} / k\right)^{2}\left(t / t_{0}\right)} .
$$

The transformation $P_{\text {SES flat }} \rightarrow P_{\text {SES cyl }}$ is useful to verify equation (30) experimentally. The transformation $P_{\text {SES cyl }} \rightarrow P_{\text {SES flat }}$ is needed in the next section to analyse LD data collected on a cylindrical sample.

$P_{\text {SES cyl }}$ is obtained from $P_{\text {SES flat }}$ by averaging and scaling the latter for the different path lengths $t(y)$ of a neutron beam crossing a cylindrical sample [see Figs. 4(a) and 4(c)]. The polarization of individual trajectories is weighted by the transmission $\operatorname{Tr}=\exp \left[-\Sigma_{k} t(x)\right]$, where $\Sigma_{k}$ and $\Sigma_{\text {th }}$ are the total absorption cross sections for neutrons with wavevectors $k$ and $k_{\text {th }}$ (th for thermal), with $k_{\text {th }}=2 \pi / \lambda_{\text {th }}$ and $\lambda_{\text {th }}=1.8 \AA$. For most isotopes there is $1 / k$ scaling if $k \leq k_{\mathrm{th}}$ :

$$
\Sigma_{k}=\frac{\Sigma_{\mathrm{th}} k_{\mathrm{th}}}{k}
$$

With the path length $t=2\left(R^{2}-y^{2}\right)^{1 / 2}$, the width of the flat sample $t_{0}$ and the cylinder radius $R$ we get

$$
P_{\mathrm{SES} \mathrm{cyl}}(z)=A^{-1} I\left[P_{\mathrm{SES} \mathrm{flat}}(z)\right]
$$

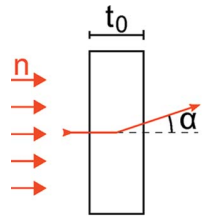

(a)

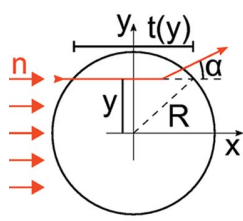

(c)

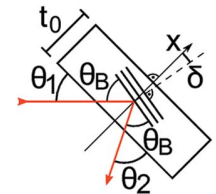

(b)

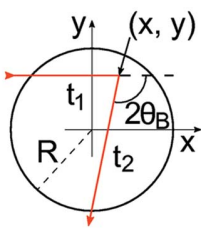

(d)
Figure 4

(a), (b) Rectangular sample. (a) In the SESANS configuration, the sample is oriented perpendicular to the incident neutron beam. A scattering event changes the direction of the neutron trajectory by an angle $\alpha .(b)$ In the LD configuration, a neutron is Bragg-diffracted at a crystallite located at a distance $x$ from the sample face. In the symmetric reflection configuration, the angles of the incident and outgoing neutron trajectories with the sample face are equal to the Bragg angle $\left(\theta_{1}=\theta_{2}=\right.$ $\left.\theta_{\mathrm{B}}\right)$. A small misalignment $\delta$ of the sample face leads to $\theta_{1}=\theta_{\mathrm{B}}-\delta, \theta_{2}=$ $\theta_{\mathrm{B}}+\delta .(c),(d)$ Cylindrical sample in $(c)$ the SESANS and $(d)$ the LD configuration. 


$$
\begin{gathered}
I\left[P_{\text {SES flat }}(z)\right] \\
=\int_{0}^{R}\left\{\left[P_{\text {SES flat }}(z)\right]^{\left(k_{0} / k\right)^{2}\left(1 / t_{0}\right)} \exp \left(-\Sigma_{k}\right)\right\}^{2\left(R^{2}-y^{2}\right)^{1 / 2}} \mathrm{~d} y, \\
A=\int_{0}^{R} \exp \left[-2 \Sigma_{k}\left(R^{2}-y^{2}\right)^{1 / 2}\right] \mathrm{d} y .
\end{gathered}
$$

Calculating $P_{\mathrm{SES} f l a t}$ from $P_{\mathrm{SES} \text { cyl }}$ data corresponds to an inversion of equation (32). This is easily achieved numerically by searching for solutions for $P_{\mathrm{SES} f l a t}(z)$ that satisfy the equation

$$
A^{-1} I\left[P_{\mathrm{SES} f l a t}(z)\right]-P_{\mathrm{SES} \text { cyl }}(z)=0 .
$$

$A$ and $I$ are the same as in equation (32). Suitable algorithms for finding roots of nonlinear functions, such as fzero() in MATLAB (The MathWorks Inc., Natick, MA, USA), are available in many numerical libraries.

\section{Scaling of SESANS data to the LD geometry}

In this section, we will determine the integrals $P_{S 1,2}$ in equation (12) from the previously measured SESANS data, for both flat and cylindrical sample geometries. For the flat sample, there are two symmetric configurations, reflection and transmission, with the sample surface perpendicular and parallel to $\mathbf{Q}$, respectively. As the effective path length in a flat sample of width $t_{0}$ at the Bragg angle $\theta_{\mathrm{B}}$ is $L_{\mathrm{r}}=t_{0} / \sin (\theta)$ in reflection and $L_{\mathrm{t}}=t_{0} / \cos (\theta)$ in transmission, $L_{\mathrm{t}}>L_{\mathrm{r}}$ for $\theta>45^{\circ}$. For the LD experiments we used $\theta=55^{\circ}$, such that $L_{\mathrm{t}} / L_{\mathrm{r}} \simeq 1.4$, leading to increased SANS in the transmission configuration. This configuration is unfavourable in our case and we will not include it in the following discussion.

$P_{S 1}, P_{S 2}$ [equation (12)] and $P_{\mathrm{SES}}$ [equation (25)] are very similar expressions, if the field inclination angles $\Omega$ in both SESANS and LD are set to $\Omega=90^{\circ}-\theta_{\mathrm{B}}$, so that $\tan (\Omega)=$ $\cot \left(\theta_{\mathrm{B}}\right)$. However, the ranges of SANS angles $S(\alpha)$ in SESANS and $S_{1,2}\left(\alpha_{1,2}\right)$ in LD are not equal, as the geometries for the two experimental configurations are different, and thus the effective neutron path lengths inside the sample are not the same.

In the SESANS configuration [Fig. 4(a)], the faces of the flat sample are aligned perpendicular to the beam. The effective neutron path length corresponds to the sample width $t_{0}$. Small variations in the neutron path length resulting from finite beam collimation $\alpha_{\mathrm{T}} \simeq 1^{\circ}$ and from small-angle scattering of the order of $t_{0} / t \simeq 1-\cos \left(\alpha_{\mathrm{T}} / 2\right)$ can be neglected in equation (30).

In the LD configuration, the path lengths vary with the location of the diffraction event. For a symmetric setup in reflection geometry as shown in Fig. $4(b)$, the path lengths of the incident and diffracted neutron trajectories are equal, with $t_{1,2}(x)=x / \sin \left(\theta_{1,2}\right)$. We obtain the polarization $P_{S 1}, P_{S 2}$ by averaging and scaling the SESANS polarization $P_{\text {SES flat }}\left(z, k_{0}, t_{0}\right)$ measured for a flat sample of width $t_{0}$ according to equation (30). The weight of an individual neutron trajectory is given by the transmission $\operatorname{Tr}_{1,2}(x)=$ $\exp \left[-\Sigma_{k} t_{1,2}(x)\right]$.

$$
\begin{aligned}
P_{S 1,2}(k, z) & =\frac{1}{B} \int_{x=0}^{t_{0}}\left\{P_{\mathrm{SES} f l a t}^{\left(k_{0} / k\right)^{2}\left[1 / t_{0} \sin \left(\theta_{1,2}\right)\right]} \exp \left[-\frac{\Sigma_{k}}{\sin \left(\theta_{1,2}\right)}\right]\right\}^{x} \mathrm{~d} x \\
= & \frac{1}{B} \frac{A^{t_{0}}-1}{\ln (A)} \\
A & =P_{\mathrm{SES} \mathrm{flat}}^{\left(k_{0} / k\right)^{2}\left[1 / t_{0} \sin \left(\theta_{1,2}\right)\right]} \exp \left[-\frac{\Sigma_{k}}{\sin \left(\theta_{1,2}\right)}\right] \\
B & =\frac{\sin \left(\theta_{1,2}\right)}{\Sigma_{k}}\left\{1-\exp \left[-\frac{\Sigma_{k} t_{0}}{\sin \left(\theta_{1,2}\right)}\right]\right\}
\end{aligned}
$$

In symmetric reflection geometry with $\theta_{1}=\theta_{2}$, we get $P_{S 1}=$ $P_{S 2}$. A detuning $\delta$ as shown in Fig. 4(b) increases $t_{1}(x)$ and decreases $t_{2}(x)$, so that the product $P_{S 1} \times P_{S 2}$ changes in second order in $\delta$, and a small detuning of the order of a few degrees is not significant.

The cylindrical sample geometry [Figs. $4(c)$ and $4(d)$ ] is treated in a similar way by taking the average of the SESANS polarization $P_{\mathrm{SES} \text { flat }}\left(z, k_{0}, t_{0}\right)$ with the transmission as a weight factor. In the case of a cylindrical sample, the SESANS data should be measured on the same sample. Then $P_{\mathrm{SES} \text { cyl }}$ has to be converted to $P_{\text {SES flat }}\left(z, k_{0}, t_{0}\right)$ [equation (35)] with a specific but arbitrary $t_{0}$. For a neutron diffracted at a crystallite located at $(x, y)$, the length of the incident trajectory inside a sample of radius $R$ is $t_{1}=x+\left(R^{2}-y^{2}\right)^{1 / 2}$.

$$
\begin{aligned}
& P_{S 1}=\frac{1}{C}
\end{aligned}
$$

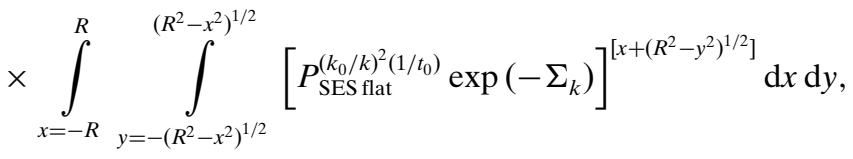

with

$$
C=\iint \exp \left\{-\Sigma_{k}\left[x+\left(R^{2}-y^{2}\right)^{1 / 2}\right]\right\} \mathrm{d} x \mathrm{~d} y .
$$

The integration boundaries are the same as in equation (39). For symmetry reasons, $P_{S 2}=P_{S 1}$.

\section{Experimental}

The LD measurements were conducted on the TRISP spectrometer at the FRM II. TRISP was designed as a thermal three-axis spectrometer incorporating the resonant spin-echo technique for the high-resolution spectroscopy of phonons and spin excitations (Keller et al., 2002), and it also operates efficiently as a Larmor diffractometer and SESANS instrument. The primary spectrometer includes a V-cavity polarizing neutron guide, a velocity selector and a pyrolytic graphite (002) monochromator. The spin-echo precession devices and the sample are housed in a mu-metal shield with a residual 
magnetic field of $<5$ mOe to avoid parasitic spin precession. For the LD measurements, the scattering angle $2 \theta_{\mathrm{B}}$ was kept constant at $110^{\circ}$ and the incident wavevector was varied in the range $2.08<k_{\mathrm{i}}<2.66 \AA^{-1}$ to access various Bragg peaks. In $\mathrm{LD}$ mode, the RF coil rotation angles were set to $\Omega=90^{\circ}-\theta_{\mathrm{B}}$ $=35^{\circ}$ to align the field boundaries parallel to the lattice planes. The same angles $\Omega=35^{\circ}$ with opposite sign in the second precession field were used in SESANS mode.

The sample was hematite, $\alpha-\mathrm{Fe}_{2} \mathrm{O}_{3}$ (powder), as used in earlier studies (Stekiel et al., 2015; Fabrykiewicz et al., 2017). Hematite is a promising material for such studies because of the negligible incoherent neutron scattering of iron and oxygen. The powder was annealed at $1100 \mathrm{~K}$ for $5 \mathrm{~h}$ in air to

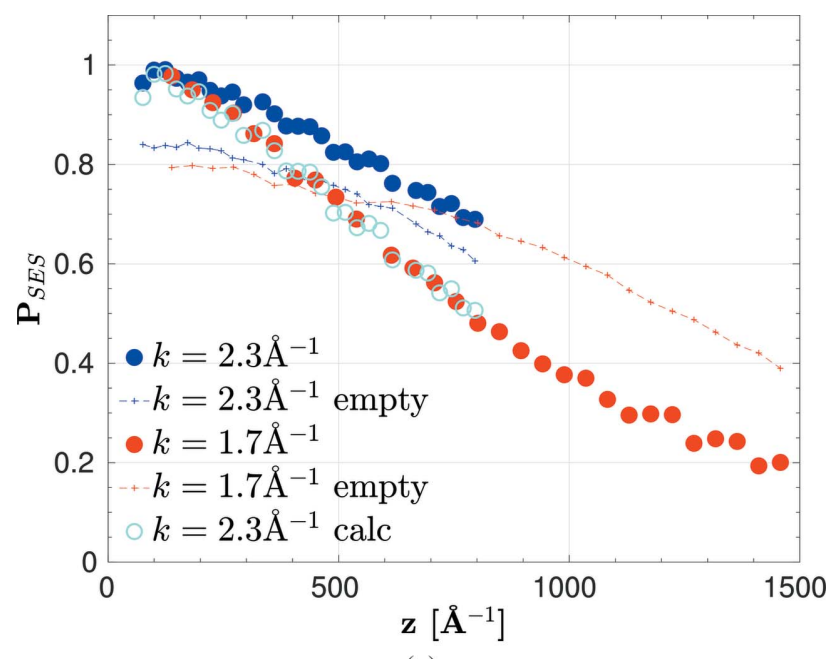

(a)

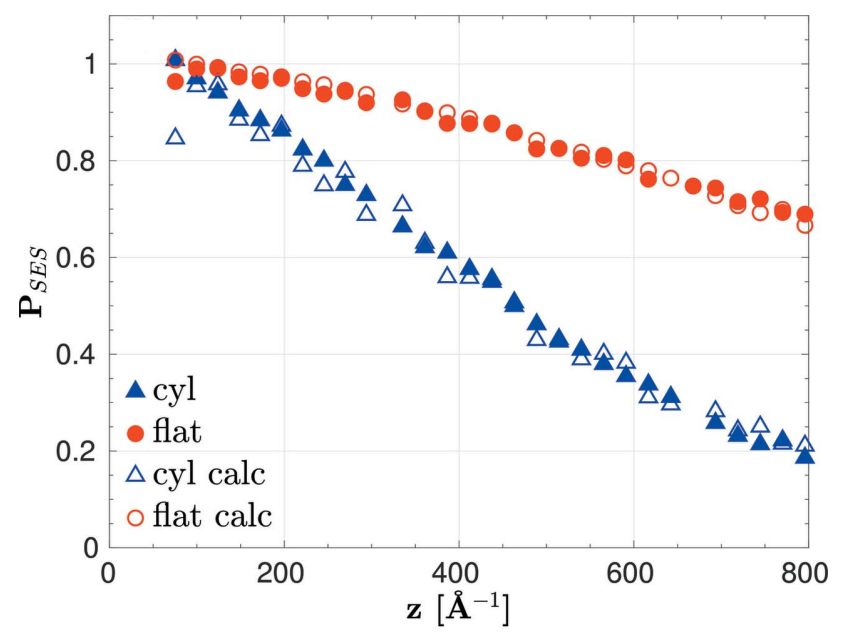

(b)

Figure 5

SESANS data for $\alpha-\mathrm{Fe}_{2} \mathrm{O}_{3}$ powder. (a) $P_{\text {SES flat }}$ for $k=1.7$ and $2.3 \AA^{-1}$ for the flat quartz glass container without sample (empty), and with sample normalized by the empty container data. The maximum $z$ for a given maximum precession field $\mathbf{B}$ scales $\sim 1 / k^{2}$. The blue open circles show the $k=2.3 \AA^{-1}$ data scaled to $k=1.7 \AA^{-1}$ by equation (30). These scaled data coincide with the experimental data for $k=1.7 \AA^{-1}$. (b) $P_{\mathrm{SES}}$ for the flat and cylindrical containers at $k=2.3 \AA^{-1}$. $P_{\text {flatcalc }}$ is the polarization calculated for the flat sample geometry by taking the $P_{\text {SEScyl }}$ data [equation (35)], and vice versa for $P_{\text {SES cylcalc }}$ [equation (32)]. The data for the cylindrical (flat) container are plotted as blue triangles (red circles). Solid symbols show measured data, while open symbols are calculations. The error bars in the measured values are smaller than the symbols.
Table 1

Experimental data on $\alpha-\mathrm{Fe}_{2} \mathrm{O}_{3}$ powder.

LD experiments were performed using four Bragg peaks. The flat container was set to reflection geometry.

\begin{tabular}{llllll}
\hline No. & $k\left(\AA^{-1}\right)$ & $(h k l)$ & Mode & Container & Contents \\
\hline 1 & 2.082 & $(024)$ & LD & Flat, reflection & Filled \\
2 & 2.082 & $(024)$ & LD & Cylindrical & Filled \\
3 & 2.263 & $(116)$ & LD & Flat, reflection & Filled \\
4 & 2.263 & $(116)$ & LD & Cylindrical & Filled \\
5 & 2.581 & $(214)$ & LD & Flat, reflection & Filled \\
6 & 2.581 & $(214)$ & LD & Cylindrical & Filled \\
7 & 2.639 & $(300)$ & LD & Flat, reflection & Filled \\
8 & 2.639 & $(300)$ & LD & Cylindrical & Filled \\
9 & 1.70 & & SESANS & Flat & Empty \\
10 & 1.70 & & SESANS & Flat & Filled \\
11 & 2.30 & & SESANS & Flat & Empty \\
12 & 2.30 & & SESANS & Flat & Filled \\
13 & 2.30 & & SESANS & Cylindrical & Empty \\
14 & 2.30 & & SESANS & Cylindrical & Filled \\
\hline
\end{tabular}

reduce internal strain. This annealing step reduced $\epsilon_{h k l}$ by $\sim 30 \%$. We used two sample cells, a cylindrical vanadium container (labelled 'cylinder'), inner diameter $12 \mathrm{~mm}$ and wall thickness $0.2 \mathrm{~mm}$, and a flat quartz glass cell (labelled 'flat'), inner dimensions $30 \times 30 \times 2 \mathrm{~mm}$ and $0.5 \mathrm{~mm}$ glass width. The flat cell produced an unstructured background (glass peak) in the LD measurement. LD was measured for (024), (116), (214) and (300) at the $k_{\mathrm{i}}$ values given in Table 1 using both cylindrical and flat containers.

For the SESANS experiments, the scattering angle was set to zero and the neutron beam was shaped by a cadmium mask to the sample size. $P_{\mathrm{SES}}$ was measured for both empty and filled sample cells at $k=2.30 \AA^{-1}$, which is roughly in the centre of the $k$ range used for $\mathrm{LD}$, and in addition at $k=$ $1.70 \AA^{-1}$ for the flat sample to test the scaling with $k$ according to equation (30). All measurements are shown in Fig. 5, where the data with sample were normalized to the empty cell measurements. (See also Table 1 for a summary of all data collected on $\alpha-\mathrm{Fe}_{2} \mathrm{O}_{3}$ ).

In LD mode, first the instrumental resolution $P_{\text {instr }}\left(\phi_{\mathrm{LD}}\right)$ was determined with a $\mathrm{Ge}$ monocrystal as reference sample with a negligible range of $d_{h k l}$, where for a perfect instrument we expect $P_{\text {instr }}=1$. The instrument configuration for this reference measurement was the same as for the subsequent LD experiment on the powder samples, with fixed $2 \theta=110^{\circ}$ and $\Omega=35^{\circ}$. The Ge (220) and (004) Bragg reflections were accessible at $k=1.92$ and $2.72 \AA^{-1}$, respectively. Fig. 6 shows these data for two different operation modes, where one $(\mathrm{RFM}=4)$ or both $\mathrm{RF}$ coils $(\mathrm{RFM}=8)$ in the individual coil sets C1-C4 (Fig. 1) were energized. These two modes differ in the effective Larmor frequency, which is two (four) times the RF for RFM $=4$ (8), and they are used to cover the range of low (high) Larmor phase. The $k$ dependence of $P_{\text {instr }}$ is a property of the polarizing neutron guide. The dependence on frequency and RFM, especially the scatter of the data points at low frequency, results from field inhomogeneities in the RF coils and from stray fields in between the coils.

Fig. 7 shows the LD data for the (024) reflection of $\alpha-\mathrm{Fe}_{2} \mathrm{O}_{3}$ measured at $k=2.08 \AA^{-1}$ for the flat container (solid lines) 


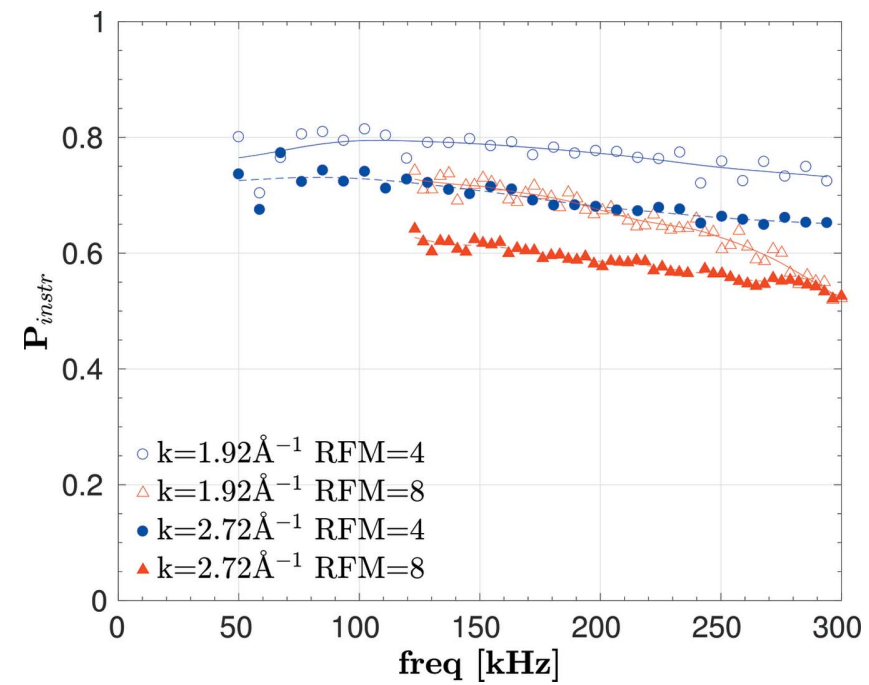

Figure 6

Instrumental resolution in LD mode measured on a Ge monocrystal using the (220) and (004) reflections with $k=1.92 \AA^{-1}$ and $k=2.72 \AA^{-1}$, respectively. The scattering angle was $110^{\circ}$ and the coil inclination angle $\Omega=35^{\circ}$. RFM $=4(8)$ is the number of active RF coils. The lines are obtained by smoothing the data. Error bars are smaller than the symbols.

and the cylindrical container (dotted lines). Data for the other Bragg peaks are shown in the supporting information. The SANS contribution $P_{S}$ is much smaller for the flat container. Normalizing the raw data by $P_{S}$ and by the instrumental polarization $P_{\text {instr }}$ (identical for both containers) leads to very similar intrinsic $P_{\mathrm{LD}}$.

The results of LD measurements for $\alpha-\mathrm{Fe}_{2} \mathrm{O}_{3}$ are compared with the results obtained by Stekiel et al. (2015) on the highresolution SR powder diffractometer ID22 at the ESRF (Fitch, 2004). The observed SR diffraction peak shape was described using a pseudo-Voigt function. The instrumental contribution of ID22 was estimated by measuring a reference $\mathrm{LaB}_{6}$ sample.

\section{Results and discussion}

In this section we discuss the analysis of LD data from $\alpha-\mathrm{Fe}_{2} \mathrm{O}_{3}$ powder. The analysis steps include (i) determination of the instrumental resolution by means of a monocrystal reference sample, (ii) measurement of the small-angle scattering by the SESANS technique, (iii) scaling these data to the LD geometry including absorption and (iv) determination of $D\left(\epsilon_{h k l}\right)$.

In LD, SESANS and other neutron spin-echo techniques, the measured signal, that is the polarization versus the Larmor precession phase, is a product of intrinsic and instrumental effects. This is in contrast with conventional diffraction and spectroscopy, where the measured signals are convolutions of the instrumental resolution and sample-related effects (Mezei, 1980; Mezei et al., 2003). This is explained, in brief, by the fact that the polarization is a probability, namely the expectation value of the neutron spinor. The probabilities of instrumental effects, such as non-perfect RF coils, and sample intrinsic effects, such as the range of $D\left(\epsilon_{h k l}\right)$, are statistically indepen-

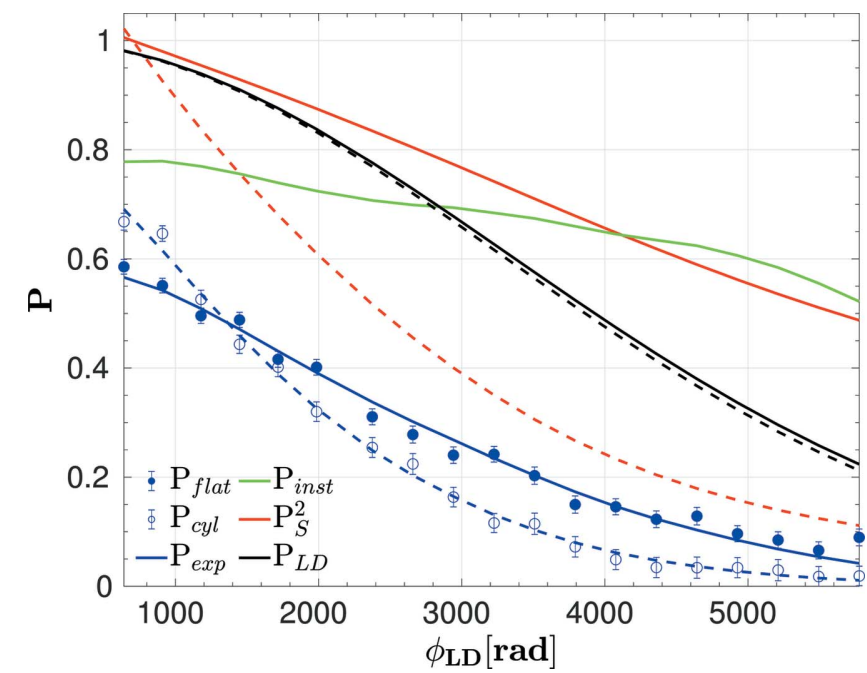

Figure 7

LD data for the (024) reflection of $\alpha-\mathrm{Fe}_{2} \mathrm{O}_{3}$ for the flat quartz glass sample container (width $2 \mathrm{~mm}$, solid symbols, solid lines) and the cylindrical vanadium container (diameter $12 \mathrm{~mm}$, open symbols, dotted lines). The counting time per point was $20 \mathrm{~min} . P_{\text {instr }}$ is the same for both containers. The fit (blue line) to $P_{\exp }$ is a product of the instrumental $P_{\text {instr }}$, SANS $P_{S}^{2}$ and $P_{\mathrm{LD}}$ [see equation (41)]. The latter depends on the range of $d_{h k l}$. A constant factor $P_{0}$ takes unpolarized background into account.

dent, so that the probabilities (polarizations) are multiplied. A second qualitative argument is that LD and spin-echo techniques provide cosine-Fourier transforms of distribution functions [equations (11) and (25)], such that convolutions are converted to products. In the present case of an LD experiment, the measured polarization $P_{\exp }$ is

$$
P_{\text {exp }}=P_{0} P_{\text {inst }} P_{S}^{2} P_{\mathrm{LD}}
$$

where $P_{S}$ and $P_{\mathrm{LD}}$ are defined in equations (12) and (17). A constant factor $P_{0}$ accounts for additional effects leading to a loss of polarization, independent of the Larmor phase, including non-polarized background from the sample and spin precession resulting from small randomly distributed ferromagnetic moments in the sample. $P_{\text {inst }}$ is taken from Fig. 6 and is independent of the sample. The same argument holds for the SESANS experiment, where

$$
P_{\text {exp SES }}=P_{0} P_{\text {inst SES }} P_{\text {SES }} \text {. }
$$

The factor $P_{S}$ in equation (41) is calculated from $P_{\operatorname{expSES}}$ in equation (42) by applying equations (36) and (39) for the flat and cylindrical samples, respectively. The instrumental resolution $P_{\text {inst }}$ is measured by means of a 'perfect' sample with no intrinsic effects, such as a monocrystal with no range of $d_{h k l}$ in the case of LD, an empty container in the case of SESANS, and a purely elastic scatterer in the case of energy-resolved spin-echo spectroscopy. In the current experiment, we used as reference samples a Ge single crystal for LD or an empty cell for SESANS. $P_{\text {inst }}($ freq, RFM, $k$ ) from the Ge crystal is shown in Fig. 6. The polarization depends on the frequency, the number RFM of energized RF coils, and the wavevector $k$. We use the smoothed curves in Fig. 6 to average the small parasitic oscillations, and a linear interpolation in $k$ between the two data sets taken at $k=1.92$ and $2.72 \AA^{-1}$. For the following 
analysis, $P_{\text {inst }}$ (freq, RFM, $k$ ) has to be converted to $P_{\text {inst }}\left(\phi_{\mathrm{LD}}\right)$, shown as a line in Fig. 7. This conversion is ambiguous, as there are two data sets with different RFMs for $P_{\text {inst }}$. Thus, we always use the data with the same RFM as used for the LD measurement $P_{\exp }$ of the powder sample.

Fig. 5 shows SESANS data normalized by the corresponding empty cell scans to eliminate instrumental effects [equation (42)], such as SANS in the coils close to the sample and non-ideal performance of the RF coils. The width $\alpha_{S}$ (FWHM) for the small-angle distributions obtained by fitting the Gaussian model equation (26) to the normalized data gives $0.0650(5), 0.1138(5)$ and $0.1427(5)^{\circ}$ for the flat cell at $k=2.3$ and $1.7 \AA^{-1}$ and for the cylindrical cell at $2.3 \AA^{-1}$, respectively. The parameter $\alpha_{S}$ depends strongly on $k$ and on the sample width, but is still much smaller than the angular transmission of the instrument $\alpha_{T} \simeq 1^{\circ}$, so that the latter has no effect in equation (26).

The LD experiment is usually performed at many Bragg reflections, each with a different $k$. The SESANS measurement should at least be done at the lowest of these $k$ values. Scaling of the SESANS data to other $k$ is possible using equation (30), as is demonstrated in Fig. 5(a) by scaling the $k=$ $2.3 \AA$ data to $k=1.7 \AA$. These scaled data coincide with the measured data. Scaling of SESANS data between flat and cylindrical sample shapes by equations (32) and (35) also works, as is shown in Fig. 5(b), where again the scaled and measured data coincide. The absorption of the $\alpha-\mathrm{Fe}_{2} \mathrm{O}_{3}$ powder was included in these plots, although it is small and has no visible effect $\left(\sigma_{\mathrm{th}}=0.10 \mathrm{~cm}^{-1}\right.$ for bulk $\alpha-\mathrm{Fe}_{2} \mathrm{O}_{3}$; the powder density is roughly $40 \%$ less). The scaling relation (30) might not hold for unconventional SANS processes, such as multiple Bragg scattering (MBS), which might contribute significantly to the SANS cross section (Barker \& Mildner, 2015). Our analysis technique is also valid for such unconventional SANS processes, if the scaling equation (30) holds. The latter can be experimentally tested by taking SESANS data at several different $k$ values.

The LD data for a (024) reflection $\left(k=2.08 \AA^{-1}\right)$ of the $\alpha-\mathrm{Fe}_{2} \mathrm{O}_{3}$ powder are shown in Fig. 7. This peak was measured at the smallest $k=2.08 \AA^{-1}$ and thus has the largest SANS contribution. $P_{S}$ shows a more pronounced decay for the cylinder due to the increased path length and increased SANS of the cylinder compared with the flat sample. The raw data without any corrections correspond to $\epsilon_{024 \text { raw }}$ values of $13.5(5) \times 10^{-4}$ for the cylinder and $8.80(11) \times 10^{-4}$ for the flat container. Including all corrections, $P_{\mathrm{LD}}$ is obtained from a fit to equation (17), corresponding to $\epsilon_{024}$ of $7.1(2) \times 10^{-4}$ and $7.2(4) \times 10^{-4}$ for the flat and cylindrical samples, respectively. (The errors are statistical errors from the fit.) This means that, after correction, the values for flat and cylindrical samples agree, where for the flat sample the SANS correction is much smaller. The polarization offsets $P_{0}$ are 0.89 (1) for the cylinder and 0.74 (1) for the flat cell, indicating the increased background from the flat quartz glass container. Data for the other Bragg peaks are shown in the supporting information.

The flat sample shape is superior to the cylinder, since for a given beam diameter (typically $20 \mathrm{~mm}$ ) a larger sample volume at a smaller width can be used. As a rule of thumb, the SANS should be limited to $P_{\mathrm{SES}} \gtrsim 0.5$ for the maximum $z$ and minimum $k$, as the relative error usually increases with decreasing $P$. This condition is roughly met in the present experiment for the flat sample, but not for the cylinder, although the sample mass is the same in both cases. The only disadvantage of the flat sample is an additional decay of the polarization in the case of an offset $\delta$ [Fig. 4(b)] from the symmetric reflection geometry. For the present data, we numerically calculated that for $\delta<10^{\circ}$ the variation $\delta \epsilon_{024}<$ 0.07 , i.e. only $1 / 3$ of the statistical error. These errors can be avoided by a precise alignment of the sample cell.

In Fig. 8 the $\epsilon_{h k l}$ obtained by LD for four Bragg peaks (flat sample) are compared with the $d$-spacing distribution widths observed on ID22. The latter were determined by numerical deconvolution of the instrumental resolution function $\left(\mathrm{LaB}_{6}\right.$ reference) from the observed raw-data Bragg peaks. The agreement of SR diffraction with LD confirms our earlier findings about the monoclinic deformation of the $\alpha-\mathrm{Fe}_{2} \mathrm{O}_{3}$ lattice (Przeniosło et al., 2014). The widths calculated for the monoclinic structure model (Przeniosło et al., 2014) are $\epsilon_{024}=$ $2.22 \times 10^{-4}, \epsilon_{116}=1.67 \times 10^{-4}, \epsilon_{214}=0.95 \times 10^{-4}$ and $\epsilon_{300}=$ $1.12 \times 10^{-4}$. The measured values are much larger, but their variation follows the variation of the calculated values. The increased width is probably due to microstrains in the powder grains.

The measured $\epsilon_{h k l}$ are close to the resolution limit of SR diffraction. For LD, it is not straightforward to define the resolution. Variations in $\epsilon_{h k l}$ are detected as a change in the polarization $P_{\mathrm{LD}}$. In typical experiments with monocrystalline samples without SANS, the error in $\epsilon_{h k l}$ is close to $10^{-5}$. The situation is more complicated for powder samples, where in the case of the flat container at the (024) reflection SANS contributes a broadening of $1 \times 10^{4}$ to $\epsilon_{h k l}$. Thus, the statistical

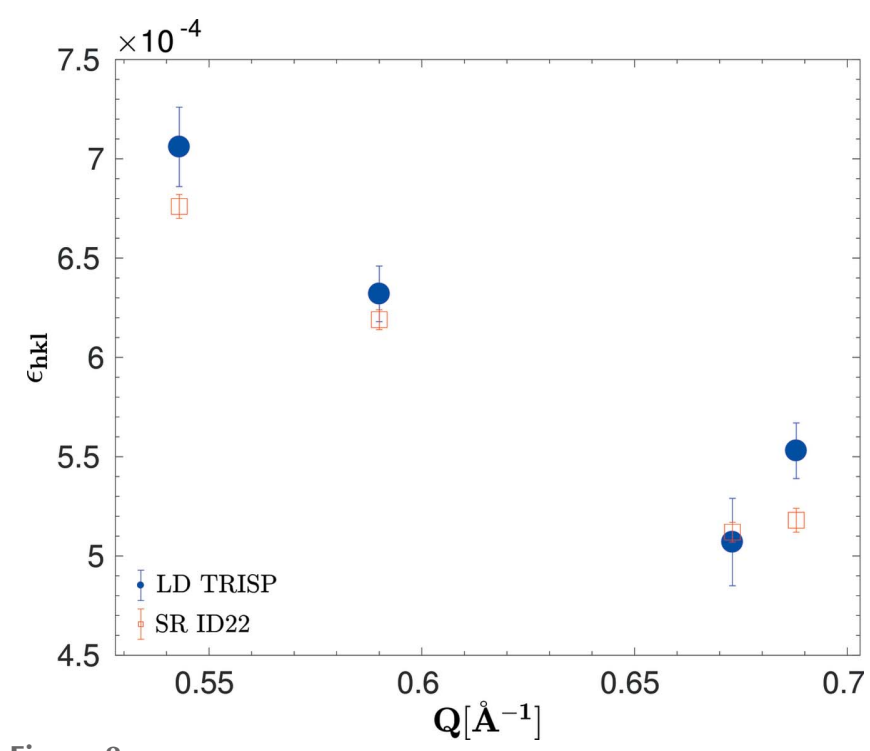

Figure 8

$\Delta d / d$ spacing distribution width $\epsilon_{h k l}(\mathrm{FWHM})$ versus $Q=1 / d_{h k l}$ for four Bragg peaks of $\alpha-\mathrm{Fe}_{2} \mathrm{O}_{3}$ obtained from LD (flat container) and SR diffraction. The data correspond from left to right to the reflections (024), (116), (214) and (300). 
errors of the order of $\pm 1.5 \times 10^{-4}$ shown in Fig. 8 as a result of the fit are also a good estimate for the resolution. The good agreement between LD and SR diffraction validates the related data analysis techniques, i.e. the measurement of the instrumental resolution with the $\mathrm{LaB}_{6}$ reference sample in SR diffraction and the determination of the instrumental resolution by a monocrystal and the correction for SANS in the case of LD.

\section{Summary}

In this work we have shown that small-angle scattering makes a significant contribution to the range of $d_{h k l}$ measured by neutron Larmor diffraction. We have described a quantitative procedure to measure the SANS by means of the spin-echo small-angle neutron scattering technique, and derived scaling expressions to include the SESANS data in the analysis of the LD data collected on flat or cylindrical samples. We have shown that using a flat sample in reflection geometry is preferable to using cylindrical samples, as for a given sample mass the effective neutron path length, and thus the effect of SANS, is minimized for the flat sample. Finally, we have applied the new analysis method to LD data from hematite and calcite powder (for the latter see the supporting information). These LD data confirm the $h k l$-dependent $d$-spacing range previously observed by high-resolution SR diffraction.

\section{APPENDIX $A$}

\section{Second-order terms of $\phi_{\mathrm{LD}}$}

The preceding discussion was based on the first-order expansion [equation (8)] of $\phi_{\mathrm{LD}}$ in equation (7). Here, we show that the second-order terms of this expansion are negligible for the analysis of the experimental data in the present work.

The expansion of equation (7) to second order in $\alpha_{1}, \alpha_{2}, \eta$ and $\epsilon_{h k l}$ gives

$$
\begin{aligned}
\phi_{\mathrm{LD}}= & \phi_{\mathrm{LD}}^{\circ}\left(1+\epsilon_{h k l}\right)-\frac{\phi_{\mathrm{LD}}^{\circ}}{2}\left(\alpha_{1}+\alpha_{2}\right) \cot \left(\theta_{0}\right) \\
& +\frac{\phi_{\mathrm{LD}}^{\circ}}{2} \sum_{i j} M_{i j} X_{i} X_{j},
\end{aligned}
$$

with the parameter vector $\mathbf{X}=\left(\alpha_{1}, \alpha_{2}, \eta, \epsilon_{h k l}\right)$ and the coefficients

$$
\mathbf{M}=\left(\begin{array}{cccc}
-\frac{1}{2} & -1-\cot ^{2}\left(\theta_{0}\right) & -\cot ^{2}\left(\theta_{0}\right) & -\cot \left(\theta_{0}\right) \\
0 & \frac{1}{2}+\cot ^{2}\left(\theta_{0}\right) & 2+3 \cot ^{2}\left(\theta_{0}\right) & -\cot \left(\theta_{0}\right) \\
0 & 0 & 1+2 \cot ^{2}\left(\theta_{0}\right) & 0 \\
0 & 0 & 0 & 0
\end{array}\right) .
$$

The polarization in equation (41) gains an additional factor $P_{\text {SO }}$ from the second-order terms:

$$
P_{\mathrm{SO}}=\prod_{i j} P_{i j \mathrm{SO}},
$$

with

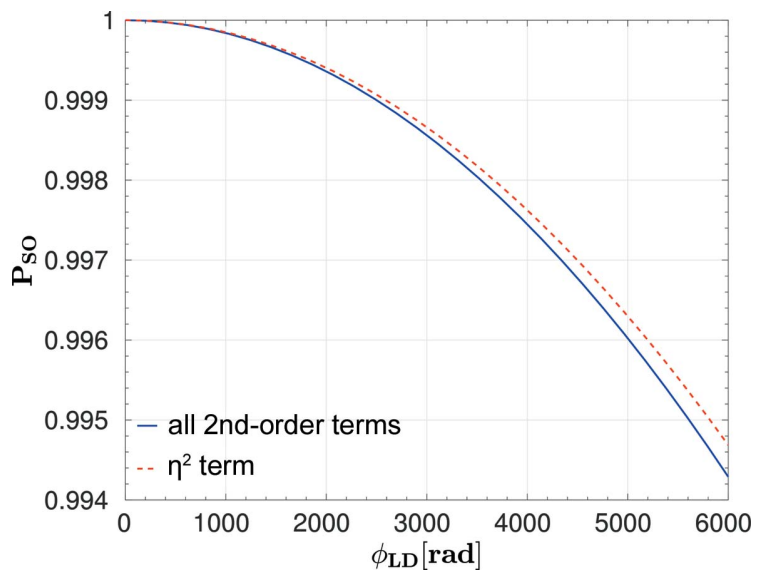

Figure 9

Second-order terms of $\phi_{\mathrm{LD}}$ [equation (46)] and the dominant $\eta^{2}$ term $P_{33 \text { so. }}$

$$
P_{i j \mathrm{SO}}=\iint F_{i}\left(X_{i}\right) F_{j}\left(X_{j}\right) \cos \left(\frac{\phi_{\mathrm{LD}}^{\circ}}{2} M_{i j} X_{i j}\right) \mathrm{d} X_{i} \mathrm{~d} X_{j} .
$$

$F_{i}\left(X_{i}\right)$ are Gaussian distribution functions for the parameters $X_{i}$, with FWHM $\alpha_{S 1}=\alpha_{S 2}=0.09^{\circ}, \epsilon_{\mathrm{FW}}=7 \times 10^{-4}$ and $\eta_{\mathrm{FW}}=$ $0.33^{\circ}$. This last parameter was obtained from the width of the rocking scan of a single perfect crystal with the same spectrometer configuration as used for LD. $\eta_{\mathrm{FW}}$ is smaller than the collimation $\left(\sim 1^{\circ}\right)$, as the wavelength bands of the incident and diffracted beams are limited by the monochromator and analyser, and thus the range of Bragg angles for a given $d_{h k l}$ is limited. Calculation of $\eta_{\mathrm{FW}}$ is in principle possible, but a suitable resolution formalism for Larmor diffractometers taking all spectrometer parameters into account, similar to the work of Habicht et al. (2003) and Groitl et al. (2018) for threeaxis spin-echo spectrometers, is not yet available.

The second-order terms in equation (43) are very small, except the $\eta^{2}$ term $\left(\sim M_{33} \eta^{2}\right)$. Fig. 9 shows the polarization equation (46) including all terms, and the $\eta^{2}$ term $P_{33}$ so separately, for the experimental parameters of this work. This dominant term changes the resulting range of $\epsilon_{h k l}=$ $7.1(4) \times 10^{-4}$ by $1.7 \times 10^{-6}$, more than one order of magnitude less than the statistical error in $\epsilon_{h k l}$. Thus we neglected this term in the present analysis. If no monochromator or analyser is used, $P_{33}$ so is large and should be included in the analysis as an additional factor in equation (41), such that

$$
P_{\text {exp }}=P_{0} P_{\text {instr }} P_{S}^{2} P_{33 \mathrm{SO}} P_{\mathrm{LD}} .
$$

\section{Acknowledgements}

We thank O. Holderer and A. Senyshyn for the sample containers, and F. Tralmer for technical support.

\section{Funding information}

We acknowledge funding from the Deutsche Forschungsgemeinschaft (DFG, German Research Foundation), Projektnummer 107745057 - TRR 80. PF acknowledges 
financial support from 'Kartezjusz' project co-financed from the European Social Fund under operational programme Knowledge Education Development, agreement No. POWR.03.02.00-00-I001/16-00.

\section{References}

Andersson, R., van Heijkamp, L. F., de Schepper, I. M. \& Bouwman, W. G. (2008). J. Appl. Cryst. 41, 868-885.

Barker, J. G. \& Mildner, D. F. R. (2015). J. Appl. Cryst. 48, 1055-1071.

Bourdarot, F., Martin, N., Raymond, S., Regnault, L.-P., Aoki, D., Taufour, V. \& Flouquet, J. (2011). Phys. Rev. B, 84, 184430.

Fabrykiewicz, P. \& Przeniosło, R. (2016). Physica B, 489, 56-62.

Fabrykiewicz, P., Przeniosło, R., Sosnowska, I. \& Fauth, F. (2018). Acta Cryst. B74, 660-672.

Fabrykiewicz, P., Stękiel, M., Sosnowska, I. \& Przeniosło, R. (2017). Acta Cryst. B73, 27-32.

Fitch, A. N. (2004). J. Res. Natl Inst. Stand. Technol. 109, 133-142.

Gähler, R. \& Golub, R. (1988). J. Phys. Fr. 49, 1195-1202.

Golub, R. \& Gähler, R. (1987). Phys. Lett. A, 123, 43-48.

Groitl, F., Keller, T. \& Habicht, K. (2018). J. Appl. Cryst. 51, 818-830.

Groitl, F., Keller, T., Quintero-Castro, D. L. \& Habicht, K. (2015). Rev. Sci. Instrum. 86, 025110.

Habicht, K., Keller, T. \& Golub, R. (2003). J. Appl. Cryst. 36, 1307-1318.

Hu, D., Lu, X., Zhang, W., Luo, H., Li, S., Wang, P., Chen, G., Han, F., Banjara, S. R., Sapkota, A., Kreyssig, A., Goldman, A. I., Yamani, Z., Niedermayer, C., Skoulatos, M., Georgii, R., Keller, T., Wang, P., Yu, W. \& Dai, P. (2015). Phys. Rev. Lett. 114, 157002.

Inosov, D. S., Friemel, G., Park, J. T., Walters, A. C., Texier, Y., Laplace, Y., Bobroff, J., Hinkov, V., Sun, D. L., Liu, Y., Khasanov, R., Sedlak, K., Bourges, P., Sidis, Y., Ivanov, A., Lin, C. T., Keller, T. \& Keimer, B. (2013). Phys. Rev. B, 87, 224425.

Keller, T., Gähler, R., Kunze, H. \& Golub, R. (1995). Neutron News, 6(3), 16-17.

Keller, T., Habicht, K., Klann, H., Ohl, M., Schneider, H. \& Keimer, B. (2002). Appl. Phys. Mater. Sci. Process. 74, s332-s335.

Keller, T. \& Keimer, B. (2015). J. Large-Scale Res. Facil. 1, A37.

Li, F., Feng, H., Thaler, A. N., Parnell, S. R., Hamilton, W. A., Crow, L., Yang, W., Jones, A. B., Bai, H., Matsuda, M., Baxter, D. V., Keller, T., Fernandez-Baca, J. A. \& Pynn, R. (2017). Sci. Rep. 7, 865.
Martin, N., Regnault, L. \& Klimko, S. (2012). J. Phys. Conf. Ser. 340, 012012.

Mezei, F. (1980). Editor. Neutron Spin Echo, Lecture Notes in Physics, Vol. 128. Heidelberg: Springer-Verlag.

Mezei, F., Pappas, C. \& Gutberlet, T. (2003). Editors. Neutron Spin Echo Spectroscopy, Lecture Notes in Physics, Vol. 601. Heidelberg: Springer.

Náfrádi, B., Keller, T., Hardy, F., Meingast, C., Erb, A. \& Keimer, B. (2016). Phys. Rev. Lett. 116, 047001.

Niklowitz, P. G., Pfleiderer, C., Keller, T., Vojta, M., Huang, Y.-K. \& Mydosh, J. A. (2010). Phys. Rev. Lett. 104, 106406.

Pfleiderer, C., Böni, P., Keller, T., Rössler, U. K. \& Rosch, A. (2007). Science, 316, 1871-1874.

Przeniosło, R., Fabrykiewicz, P. \& Sosnowska, I. (2016). Physica B, 496, 49-56.

Przeniosło, R., Fabrykiewicz, P., Sosnowska, I., Wardecki, D., Sławiński, W., Playford, H., Hempelmann, R. \& Bukowski, M. (2018). Physica B, 530, 183-190.

Przeniosło, R., Sosnowska, I., Stękiel, M., Wardecki, D., Fitch, A. \& Jasiński, J. B. (2014). Physica B, 449, 72-76.

Rekveldt, M. T. (1996). Nucl. Instrum. Methods Phys. Res. B, 114, 366-370.

Rekveldt, M. T. (2000). Physica B, 276-278, 55-58.

Rekveldt, M. T., Bouwman, W. G., Kraan, W. H., Uca, O., Grigoriev, S. V., Habicht, K. \& Keller, T. (2003). Neutron Spin Echo Spectroscopy, Lecture Notes in Physics, Vol. 601, edited by F. Mezei, C. Pappas \& T. Gutberlet, pp. 87-99. Heidelberg: Springer.

Rekveldt, M. T., Keller, T. \& Golub, R. (2001). Europhys. Lett. 54, 342-346.

Repper, J., Keller, T., Hofmann, M., Krempaszky, C., Petry, W. \& Werner, E. (2010). Acta Mater. 58, 3459-3467.

Ruminy, M., Groitl, F., Keller, T. \& Fennell, T. (2016). Phys. Rev. B, 94, 174406 .

Sosnowska, I., Przeniosło, R., Palewicz, A., Wardecki, D. \& Fitch, A. (2012). J. Phys. Soc. Jpn, 81, 0446041.

Stękiel, M., Przeniosło, R., Sosnowska, I., Fitch, A., Jasiński, J. B., Lussier, J. A. \& Bieringer, M. (2015). Acta Cryst. B71, 203 208.

Wang, W., Song, Y., Cao, C., Tseng, K.-F., Keller, T., Li, Y., Harriger, L. W., Tian, W. C. S., Chi, S., Yu, R., Nevidomskyy, A. H. \& Dai, P. (2018). Nat. Commun. 9, 3128. 